\title{
Review Article \\ Hybrid Neural Network Approach Based Tool for the Modelling of Photovoltaic Panels
}

\author{
Antonino Laudani, Gabriele Maria Lozito, \\ Francesco Riganti Fulginei, and Alessandro Salvini \\ Department of Engineering, Roma Tre University, Via Vito Volterra 62, 00146 Rome, Italy \\ Correspondence should be addressed to Antonino Laudani; antonino.laudani@uniroma3.it
}

Received 19 November 2014; Revised 15 January 2015; Accepted 17 January 2015

Academic Editor: Cheuk-Lam Ho

Copyright (c) 2015 Antonino Laudani et al. This is an open access article distributed under the Creative Commons Attribution License, which permits unrestricted use, distribution, and reproduction in any medium, provided the original work is properly cited.

\begin{abstract}
A hybrid neural network approach based tool for identifying the photovoltaic one-diode model is presented. The generalization capabilities of neural networks are used together with the robustness of the reduced form of one-diode model. Indeed, from the studies performed by the authors and the works present in the literature, it was found that a direct computation of the five parameters via multiple inputs and multiple outputs neural network is a very difficult task. The reduced form consists in a series of explicit formulae for the support to the neural network that, in our case, is aimed at predicting just two parameters among the five ones identifying the model: the other three parameters are computed by reduced form. The present hybrid approach is efficient from the computational cost point of view and accurate in the estimation of the five parameters. It constitutes a complete and extremely easy tool suitable to be implemented in a microcontroller based architecture. Validations are made on about 10000 PV panels belonging to the California Energy Commission database.
\end{abstract}

\section{Introduction}

Nowadays, the photovoltaic (PV) based generation systems are extremely common and you can view both small plants (some kWpp) on the roof top or larger plants (MWpp) usually in rural or industrial environments. From the point of view of the technology and materials employed, several steps have been done in order to improve the efficiency and the performances [1]. On the other hand, from the point of view of the system, the major study has concerned the aspect of both characterization and energy conversion, giving to the designers some advanced tools for the setup of the generation system. High level performances are usually achieved by using suitable conversion systems, which try to make work the system in the maximum power point condition [2]. Although these conversion systems consist, in some case, in extremely complex processes and even in artificial intelligence based algorithms, there is a lack in studying of the effects of environmental conditions and in ageing of the parameters characterizing the PV modules; consequently, considering the PV system as a whole, some additional components would be needed which allow both the monitoring of each PV module and the intelligent management of the PV system aimed at the optimization of the performances. In particular, there is a scarcity of embedded systems able to characterize in real time the PV arrays during their normal working in order to update the parameters of the PV model for a better estimation of generated power. The reason of this lack is essentially due to two issues: (i) the requirement of several sensors for the continuous monitoring of the PV plants and (ii) the difficulty of identifying in real time the PV model, since this requires the solution of a transcendental (nonlinear) problem, the five-parameter model, which is really hard to solve without the use of suitable computing environment such as Matlab, Mathematica, and Maple. In this work, by following our previous successful experiences in the application of neural networks (NNs) to the PV field, we propose a solution of the identification problem for the five-parameter model starting from few information, thanks to the synergy between a neural network and an analytical approach, the so-called reduced forms of the one-diode model. The proposed procedure can be easily implemented in embedded algorithms based on 
low cost microcontroller, allowing the development of a new strategy for the intelligent management of PV plants. This work is structured as follows: in Section 2 a brief review of the application of soft computing to PV field is presented; the one-diode model and the problems linked to its identification are illustrated in Section 3, together with the innovative method based on reduced form; the proposed neural solution with the issues of its setup and training is described in Section 4; results and validations on real data are reported in Section 5; authors' conclusions follow in Section 6.

\section{The Application of Soft Computing Techniques to PV System}

The soft computing techniques have been currently applied in several works in the literature for the solution of different issues regarding PV systems. One of the most successful applications is the offline identification of the PV model of a PV system from measurements; almost all the soft computing based optimization techniques have been adopted to address this problem: simulated annealing [3], genetic algorithm [4], differential evolution $[5,6]$, evolutionary algorithm $[7,8]$, artificial bee swarm optimization [9], bacterial foraging algorithm [10], semianalytical/deterministic approach, and so on [11-13] (see also the references within the above cited papers). On the other hand, the same optimization techniques have been less used to face other kinds of problems regarding PV systems because of their high computational costs, which make impossible their use in real time and embedded application. Only recently, some attempts have been successfully done to PV array reconfiguration [14] and maximum power point tracking $[15,16]$. Among the soft computing techniques, neural networks and fuzzy logic, thanks to their intrinsic nature and their online low computational cost, have been successfully utilized in the PV field, often by hybrid configurations [17]. For example, controllers based on fuzzy logic have been used to face the maximum power point tracking (MPPT) [18], to manage a storage system [19], to predict daily irradiation [20], and for the PV array reconfiguration [21]. The neural networks (NNs) have been widely used in the field of renewable energy since more than 20 years (a review of year 2001 can be found in [22]) and their application to PV field still arouses interest as testified by recent papers on MPPT algorithms [2, 23-26] and on the energy production estimation and forecasting (a review was published by [27]). In the last year, we have used NNs also for the development of some components of the PV system (irradiance sensor and maximum power point tracking), implementing them in a low cost microcontroller based environment: indeed, one of the main advantages in using NNs with respect to other soft computing techniques is their easy implementation and good performance in terms of both computational costs and memory consumption [28]. However, although NNs are often applied in the literature to efficiently solve mathematical problems $[29,30]$, in the PV module modeling they are only used to interpolate experimental data rather than to identify a real model. In the opinion of the authors, this fact may be due to the difficulty in obtaining a suitable training set able to effectively represent the identification problem, but this is not the only problem affecting the neural approach, as we will show in this paper: indeed, the output parameters (in particular the shunt resistance) often present variations with respect to input data that are very difficult to establish, and no neural approach is able to perform an acceptable identification, at least with a limited number of neurons and reasonable computational costs. Nevertheless, as we will demonstrate, the use of reduced form of one-diode model helps us to overcome this problem and makes the proposed approach feasible and efficient.

\section{The One-Diode Model: Identification Problem and Its Reduced Form}

The five-parameter model, also known as "one-diode model," for the electrical representation of a solar panel is widely used in solar power industry and is generally recognized as design tool [31]. This model was originally formulated for a PV cell, but under some conditions it has been demonstrated valid for a module composed by an arbitrary number of cells and also for a generic PV array composed by series and parallel connected modules. The importance of the onediode model and its success is due to this generalization capability: it is enough accurate under the hypothesis that all the cells/modules work in the same conditions of irradiance and temperature, hypothesis usually verified with a good accuracy in a not shaded module/array. Among the different versions [32] of the five-parameter model, in this work we use the one proposed and validated by de Soto et al. [33], which is also the most adopted one. The original model is based on the circuit representation of a photovoltaic device by means of an independent current source, an antiparallel diode, and two output resistances, one in parallel with the diode and the other one in series with the output branch (see Figure 1). The model current-voltage relation is expressed by (1)

$$
I=I_{\mathrm{Irr}}-I_{0}\left[\exp \left(\frac{q\left(V+I R_{S}\right)}{N_{S} n k T}\right)-1\right]-\frac{V+I R_{S}}{R_{\mathrm{SH}}} .
$$

This equation represents a PV module (or an array of modules) composed by an arbitrary number $N_{S}$ of cells in series (or modules connected in series). The parameters (nomenclature of the parameters involved is reported in Nomenclature section) of equation (1) are the ideality factor $n$, the shunt resistance $R_{\mathrm{SH}}$, the series resistance $R_{S}$, the saturation reversal current of the diode $I_{0}$, and the irradiation current $I_{\text {Irr. }}$. The other physical quantities involved are the following: the electron charge $q=1.602 \times 10^{-19} \mathrm{C}$; the Boltzmann constant $k=1.3806503 \times 10^{-23} \mathrm{~J} / \mathrm{K}$; and the cell temperature $T$. Clearly the above parameters depend on both temperature $T$ and irradiance level $S$.

3.1. Identification of the One-Diode Model. The five-parameter model can be identified offline by fitting experimental data at an assigned temperature and irradiance, or by solving a system of five nonlinear equations starting from data provided by manufacturers in datasheets. In this last case the parameters can be derived by the following datasheet values: 


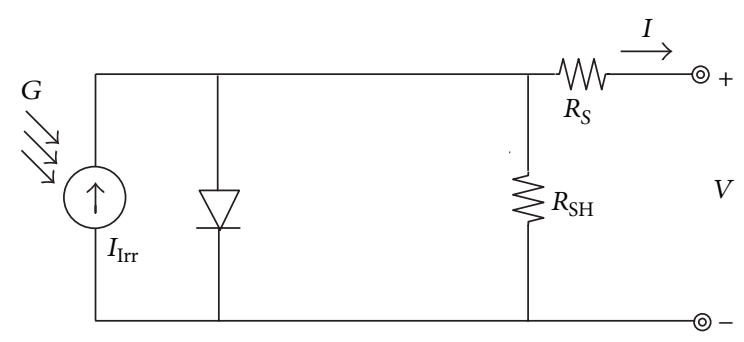

FIgURE 1: One-diode equivalent circuit of a PV module.

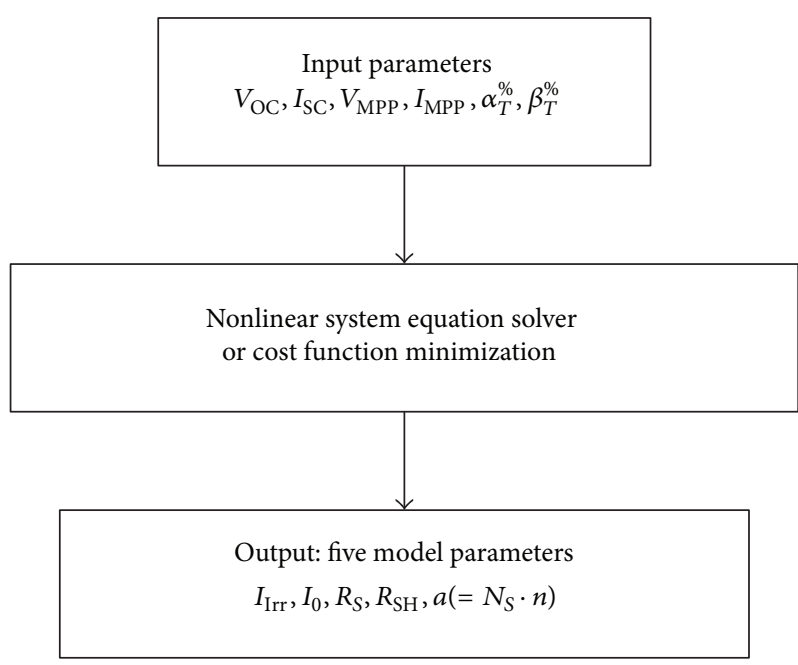

FIGURE 2: Five-parameter model identification by using the techniques illustrated in [34].

the open circuit voltage $V_{\mathrm{OC}}$, the short circuit current $I_{\mathrm{SC}}$, the voltage and current at the maximum power point $I_{\mathrm{MPP}}$ and $V_{\mathrm{MPP}}$, and the temperature coefficients $\alpha_{T}$ and $\beta_{T}$ for $I_{\mathrm{SC}}$ and $V_{\mathrm{OC}}$, respectively. By using these datasheet values, usually five implicit nonlinear equations in the unknowns of $n, R_{\mathrm{SH}}, R_{S}, I_{0}$, and $I_{\mathrm{Irr}}$ are formulated. Unfortunately, the resulting identification problem must be solved numerically by means of nonlinear solving methods or by using optimization/inverse problem algorithms in terms of minimization of a cost function (see Figure 2). In any case, the optimization problem with five unknowns can be rather long and complex to solve without a suitable computational architecture, also for the transcendental nature of the equations involved. In addition, the found results are valid at standard reference conditions (SRC) with a solar irradiance $G_{\text {REF }}=1000 \mathrm{~W} / \mathrm{m}^{2}$ and a temperature $T_{\mathrm{REF}}=25^{\circ} \mathrm{C}$; thus, the five parameters are usually indicated with the apices (or pedices) REF or SRC. As stated above, apart from the ideality factor $n$ and the series resistance $R_{S}$, the other three parameters are temperature and/or irradiance dependent. Indeed, three relations expressing the parameters for arbitrary temperature and irradiance are available [33]. Then, once the parameters are extrapolated at SRC, they can be modulated by means of these three simple relations to obtain an $I-V$ characteristic curve for any condition.
3.2. Reduced Form: A New Paradigm in the Five-Parameter Model Identification. In 2013 in [35] the issue of the fiveparameter model identification was addressed in a different way. The authors deduced that the model could be reduced by following algebraic manipulation to a two-parameter model, being three of the original parameters dependent on the other two. In particular, the unknowns $I_{\mathrm{Irr}}, I_{0}$, and $R_{\mathrm{SH}}$ can be expressed as functions of the unknowns $n$ and $R_{S}$ by means of the following exact closed forms:

$$
\begin{aligned}
R_{\mathrm{SH}}^{-1}= & h\left(n, R_{S}\right) \\
= & \frac{\operatorname{Exp}_{\mathrm{OC}}\left(I_{\mathrm{MPP}}-I_{\mathrm{SC}}\right)+\operatorname{Exp}_{\mathrm{MP}} I_{\mathrm{SC}}-\operatorname{Exp}_{\mathrm{SC}} I_{\mathrm{MPP}}}{A_{1} \operatorname{Exp}_{\mathrm{SC}}+A_{2} \operatorname{Exp}_{\mathrm{MP}}+A_{3} \operatorname{Exp}_{\mathrm{OC}}}, \\
I_{0}= & f\left(n, R_{S}\right) \\
= & \frac{V_{\mathrm{OC}}\left(I_{\mathrm{SC}}-I_{\mathrm{MPP}}\right)-V_{\mathrm{mp}, \mathrm{ref}} I_{\mathrm{SC}}}{A_{1} \operatorname{Exp}_{\mathrm{SC}}+A_{2} \operatorname{Exp}_{\mathrm{MP}}+A_{3} \operatorname{Exp}_{\mathrm{OC}}} \\
I_{\mathrm{Irr}}= & g\left(n, R_{S}\right) \\
= & \left(I_{\mathrm{SC}} V_{\mathrm{OC}}\left(\operatorname{Exp}_{\mathrm{MP}}-1\right)+I_{\mathrm{SC}} V_{\mathrm{MPP}}\left(1-\operatorname{Exp}_{\mathrm{OC}}\right)\right. \\
& \left.+I_{\mathrm{MPP}} V_{\mathrm{OC}}\left(1-\operatorname{Exp}_{\mathrm{SC}}\right)\right) \\
& \cdot\left(A_{1} \operatorname{Exp}_{\mathrm{SC}}+A_{2} \operatorname{Exp}_{\mathrm{MP}}+A_{3} \operatorname{Exp}_{\mathrm{OC}}\right)^{-1},
\end{aligned}
$$

where

$$
\begin{gathered}
A_{1}=V_{\mathrm{MPP}}+R_{S} I_{\mathrm{MPP}}-V_{\mathrm{OC}}, \\
A_{2}=V_{\mathrm{OC}}-R_{S} I_{\mathrm{SC}}, \\
A_{3}=R_{S} I_{\mathrm{SC}}-R_{S} I_{\mathrm{MPP}}-V_{\mathrm{MPP}}, \\
\operatorname{Exp}_{\mathrm{OC}}=e^{\left(V_{\mathrm{OC}} / N_{S} n V_{T}\right)}, \\
\operatorname{Exp}_{\mathrm{MPP}}=e^{\left(\left(V_{\mathrm{MPP}}+R_{S} I_{\mathrm{MPP}}\right) / N_{S} n V_{T}\right)}, \\
\operatorname{Exp}_{\mathrm{SC}}=e^{\left(R_{S} I_{\mathrm{SC}} / N_{S} n V_{T}\right)} .
\end{gathered}
$$

This formulation brings several benefits: the unknowns of the problem are reduced to two $\left(n\right.$ and $\left.R_{S}\right)$ thus allowing to use simpler optimization techniques/method to solve the problem; the reduced form makes the original problem a convex problem (one unique solution) and the resulting twoequation system constitutes an optimization problem easier to solve than the one related in the five-parameter model identification $[34,36]$; the search domain is well defined with lower and upper bounds for both of the unknowns [32]. However, the implementation of the numerical solver for the reduced form may again not be so easy without a suitable computing environment, such as Matlab and Mathcad. On the other hand, as it has been demonstrated in [32], this formulation constitutes a new paradigm for the one-diode model: indeed (2), (3), and (4) can be used instead of open circuit, short circuit, and maximum power point conditions, since any acceptable values (i.e., values belonging to the feasible domain) of the two independent unknowns $n$ and $R_{S}$ substituted in (2), (3), and (4) make these conditions 
exactly satisfied. This last feature is extremely important also in our neural approach, as it will be shown in the following section. Nevertheless, thanks to these reduced/closed forms implemented in Matlab environment, it was possible to quickly solve the identification problem and consequently to build a database of solutions related to more than 10000 modules to be used for generating the training dataset and the test dataset for our neural network identifier. In order to do this, one five-parameter model was identified by using the techniques illustrated in [34] for each module of the database [37] (see Figure 2). It is worth noticing that in order to avoid passing the number of cells in series $N_{S}$ as input the modified ideality factor parameter $a=n N_{S}$ was used as output, instead of the standard ideality factor $n$.

\section{The Neural Network Identifier}

Different possibilities arise when choosing for a NN architecture. First of all, the nature of the problem must be examined: static or dynamic problems affect the choice among feed forward or recurrent networks. In our case, the problem consists in a static identification problem which can be solved by a feed forward approach. Another point to take into account is the complexity of the implementation in a low cost/performance system. Starting from these considerations the multilayer perceptron (MLP) architecture is surely an effective choice for this kind of problems with respect to other neural network architectures such as fully connected neural networks or radial basis neural networks. In the MLP architecture the neurons are organized by layers and the connections are made using a feed forward configuration: no connection exists between neurons of the same layer and each neuron communicates via weighted connections to all the neurons of the successive layer. The number of layers in a MLP is variable, with a minimum of three: input, hidden, and output layers. The input layer simply performs the NN input connections. The hidden layer is composed by neurons with a nonlinear activation function. The output layer is analogous to the second but with simple linear activation functions. By the Universal Approximation Theorem [38, 39] a feed forward neural network with a single hidden layer containing a finite number of neurons can interpolate any continuous function. A remarkable advantage of this architecture consists in its univocal determination by the number of neurons: given the hidden layer size, the number of inputs, and the number of outputs, the NN architecture is completely defined. Another important issue is that the relation between training performance and layer size has been thoroughly investigated in the literature [40-44]. Surely complex problems may require a very high number of neurons, making the training of a single hidden layer MLP difficult: that is, even if the MLP could approximate any function, it may not be the most efficient way to do it [45]. Nevertheless, it is the first typology of NN usually attempted when a new problem is addressed for the first time. In addition we have decided to investigate the use of MLP architecture for the implementation of our neural identifier, since we have already successfully used it for other PV related problems such as for the solution of maximum power point

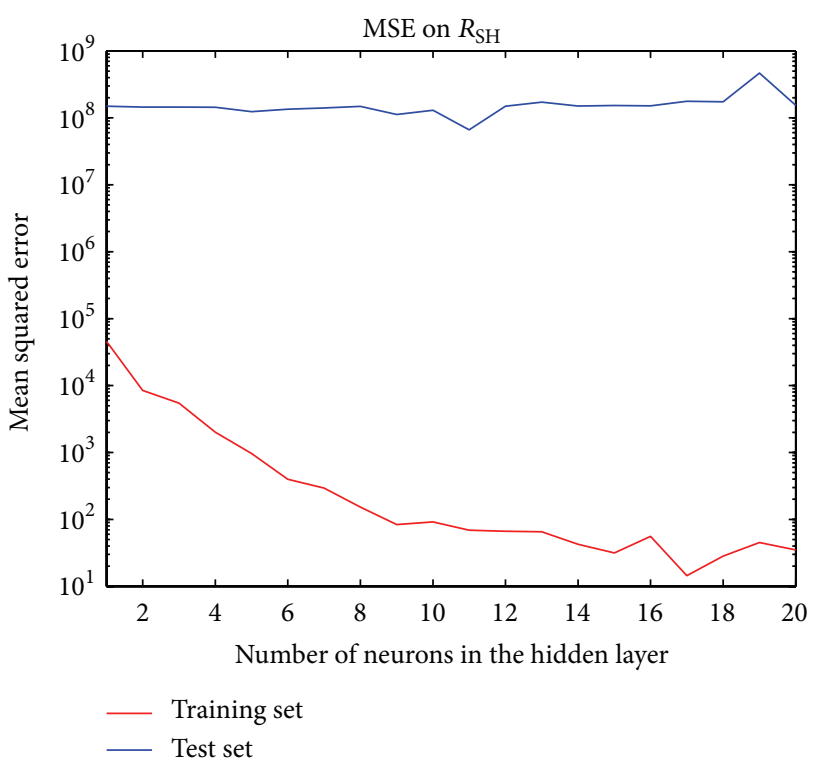

FIGURE 3: Mean squared error on training and validation set for MLP estimating $R_{\mathrm{SH}}$ parameter (hidden layer size up to 20 neurons).

identification [2] and for the prediction of solar irradiance from PV voltage and current measurements [46]. The aim this time is to use a neural network to estimate the parameters of the one-diode model starting from few available data, as explained in Section 3. The simplest strategy would be to substitute the central block shown in Figure 2 with a MLP with multiple inputs and multiple outputs (MIMO) structure, to train it on the training set, and to validate the obtained results. However, by using a MIMO architecture, an effective estimation with NN cannot be achieved for the addressed problem. To understand the reasons behind this behavior, in our study, the MIMO architecture was split into 5 MISO networks [47] (more simple to train), one for each of the five parameters figuring in the model. By trying to compute the parameters individually, the problem became apparent: the parameter $R_{\mathrm{SH}}$ may not be accurately estimated by the NN, at least by using a low/medium number of neurons, or even by directly using the MIMO network. The performance of the MISO network up to 20 neurons is shown in Figure 3; it is worth noticing that even if the performance on the training data decreases with the number of neurons, the same behaviour is not present in the performance on the test dataset: the MISO neural network is not able to generalize the prediction of $R_{\mathrm{SH}}$. This parameter, by itself, is the cause of failure of the NNs based approach. This justifies the low presence in the literature of neural approaches for the identification of PV models.

On the contrary, by taking advantage of the closed forms proposed in Section 3, the calculation of $I_{0}, R_{\mathrm{SH}}$, and $I_{\mathrm{Irr}}$ is not necessary to identify the model and the number of parameters NNs have to predict becomes just two. Indeed, as it can be seen in the block diagram proposed in Figure 4, the $\mathrm{NN}$ approach can be used to evaluate only the two independent parameters $R_{S}$ and $a$, whereas the remaining three dependent parameters can be calculated by using 


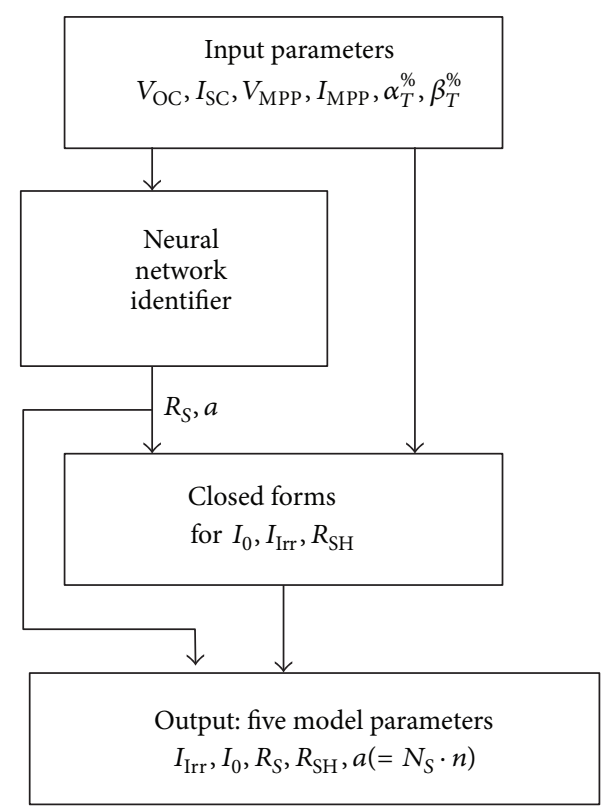

FIGURE 4: Identification of the parameters by means of a neural identifier and explicit equations.

the closed forms $h\left(n, R_{S}\right), f\left(n, R_{S}\right)$, and $g\left(n, R_{S}\right)\left(n=a / N_{S}\right)$, that is, the formulae (2), (3), and (4), respectively. Thus, two MISO NNs have been implemented: they both receive as input the six known values available on PV module datasheet (the open circuit voltage $V_{\mathrm{OC}}$, the short circuit current $I_{\mathrm{SC}}$, the voltage and current at the maximum power point $I_{\mathrm{MPP}}$ and $V_{\mathrm{MPP}}$, and the temperature coefficients $\alpha_{T}$ and $\beta_{T}$ of $I_{\mathrm{SC}}$ and $V_{\mathrm{OC}}$, resp.) and return $R_{S}$ (one $\mathrm{NN}$ ) and $a$ (the other $\mathrm{NN}$ ) as outputs.

4.1. Dataset and Training Procedure Description. The dataset used in this work to train and to validate the neural networks was obtained from the California Energy Commission (CEC) panel database [37]. The database collects the most relevant datasheet information for about 10000 photovoltaic panels. As stated above, by using the techniques illustrated in [34], for each module of the database, one five-parameter model was identified. In order to verify the generalization capabilities of our neural network, we used modules with monocrystalline silicon (mono-Si) technology as training set and modules with multicrystalline silicon (multi-Si) technology as test set. Thus, the resulting training set consists of about 4000 entries, whereas the test set has more than 6000 modules. Choosing the mono-Si modules should also guarantee a training dataset able to represent a good heterogeneity of panels with different characteristics. Clearly, the generalization capabilities and the performance of a NN for a specific problem also depend on the architecture of the $\mathrm{NN}$, on the number of neurons, on the activation function, and on some strategy adopted for the learning procedure. For example, a relationship can be achieved for each kind of problem, which links the number of neurons with the size of the training set (details can be found in [48]) for a fixed generalization level avoiding to overtrain

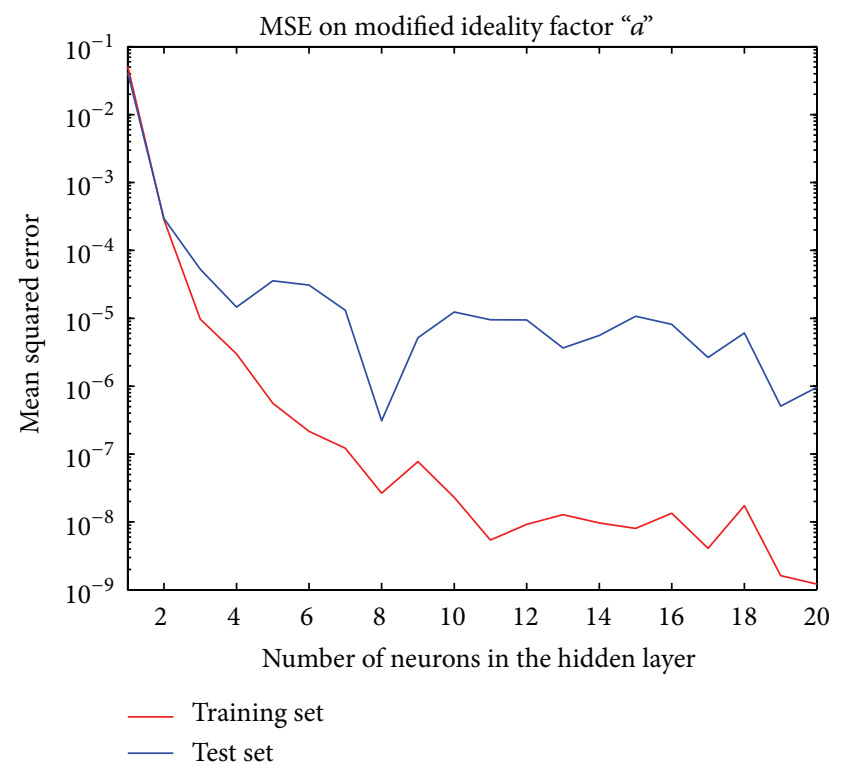

FIGURE 5: Trend of the MSE on training set and test set of the neural network tailored for predicting the $a$ parameter versus the number of neurons in the hidden layer.

the NN. In our case the training set is very large with respect to the number of adopted neurons and consequently it is unlikely to bump into overtrained networks; thus, we did not use "validation set" and "early stop technique." Rather, for our application, it is extremely important to reach high accuracy. For these reasons, the approach chosen for the training procedure consists of two phases. In the first preliminary phase we adopted the following conditions: (i) the number of neurons, $n_{\text {hidden }}$, in the hidden layer was variable from 1 up to 20; (ii) each $\mathrm{NN}$ with a fixed number of hidden layer neurons was trained 10 times for a number of epochs equaling $2000+100 \cdot n_{\text {hidden }}$ in order to obtain a statistical analysis of results (evaluation of minimum, average, median, and standard deviation of the error). The performance of the neural networks trained in terms of minimum MSE on training set and test set is reported in Figures 5 and 6 for the parameters $a$ and $R_{S}$, respectively. As it is possible to notice, the MSE reaches extremely low values (of the order of 1e-7) giving an accurate estimation of these two parameters. It is also worth reminding that the training set is made of monoSi modules whereas the test set is made of multi-Si modules: the obtained low values of MSE on test set and training set confirm the good generalization capabilities of the NNs for both of the technologies. In addition, it was verified that the MSE further decreases by adding new neurons, but the result being enough accurate this would determine only a higher computational cost.

Instead, from these results/data the two best NNs were selected and the second phase of training was started. In the choice of the best NNs we used on one hand the criterion of performance on test set and training set and on the other hand an equal size (number of neurons) of the two NNs, in such a way to be able to fruitfully compose the two NNs in 


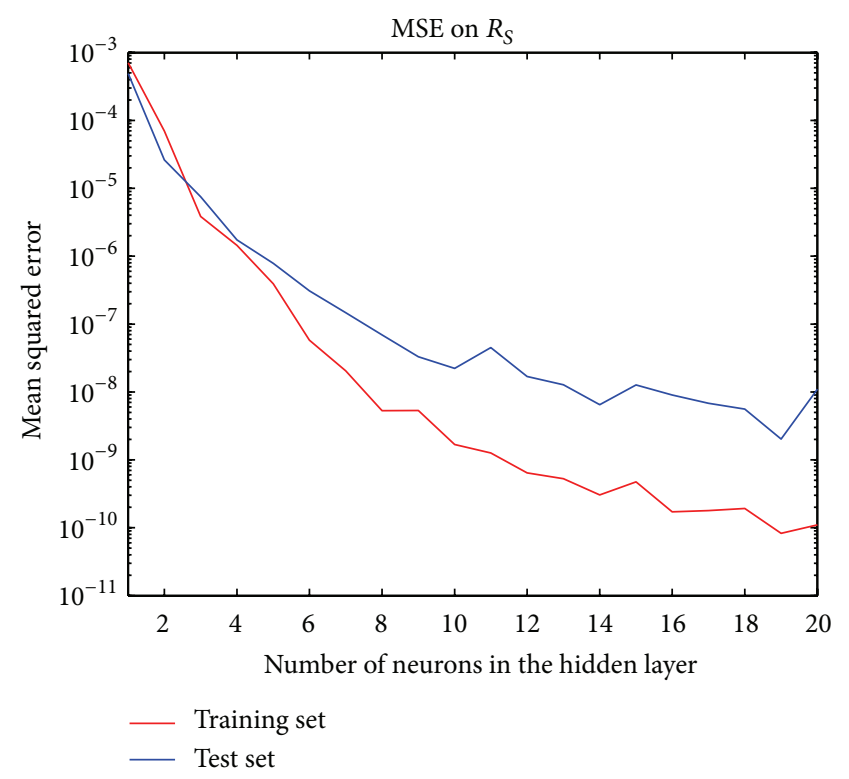

FIGURE 6: Trend of the MSE on training set and test set of the neural network tailored for predicting the $R_{S}$ parameter versus the number of neurons in the hidden layer.

the implementation (this is above all useful in the case of microcontroller implementation). Thus an effective number of neurons of hidden layer, $n_{\text {hidden }}^{*}=19$, were individuated for the two NNs. A schematization of the NNs with the used activation functions is shown in Figure 7. Then, in the second phase, these two NNs were trained, starting from the achieved weights and bias (by using the same training set) for 50000 epochs: Figure 8 shows the MSE during this second training of the neural network tailored for computing the $a$ parameter. Now, with 50000 epochs, the MSEs achieved by NNs on test set are of the order of $10^{-9}$ for both $a$ and $R_{S}$ parameters.

\section{Validation of the Proposed Neural Approach and Discussion of the Results}

Since our aim is to identify all five parameters, it is important to also verify the error achieved in the remaining three parameters when the approach described before (summarized in the block diagram of Figure 4) is used. The goodness of achieved results in terms of mean relative error in \% on test set (more than 6000 modules) is reported in Table 1. For all the parameters the error is lower than $1 \%$ and the parameter which presents the highest relative error is $I_{0}$ : this is clearly due to the fact that it assumes values close to zero (of the order of $1 \mathrm{E}-9$ ) and then the relative error is not the best way to evaluate the performance in the computation.

As said before, although the accuracy of the obtained parameters could be enhanced by adding further neurons, this is not necessary for two different reasons. Firstly, in every case, it is already quite acceptable, considering that the state of the art for this computation often utilized approximate formulae introducing errors of the order of $1 \%$ (see $[49,50])$. At the same time, the other three dependent parameters
TABLE 1: Mean relative error in $\%$ on test set.

\begin{tabular}{lc}
\hline Parameter & Mean absolute error \\
\hline$a$ & $0.00053 \%$ \\
$R_{S}$ & $0.006 \%$ \\
$I_{\text {Irr }}$ & $0.057 \%$ \\
$I_{0}$ & $0.48 \%$ \\
$R_{\mathrm{SH}}$ & $0.13 \%$ \\
\hline
\end{tabular}

$R_{\mathrm{SH}}, I_{0}$, and $I_{\text {Irr }}$ being computed by using the respective closed form expressions previously presented, they surely satisfy the open circuit, short circuit, and maximum power point conditions. This last property also demonstrates the importance of using a hybrid approach between the neural model and the closed forms (reduced forms of five-parameter model). In order to better show this important feature, we propose herein the results in terms of $I-V$ and $P-V$ curves drawn starting from the parameters calculated with the proposed approach. These results are relative to a PV module manufactured by Kyocera, the PV Panel KD210GX LP. Figure 9 shows the $I-V$ curve, whereas Figure 10 shows the $P-V$ curve. As it is possible to see from Figure 9 the $I-V$ curve exactly passes through the points of short circuit, open circuit, and maximum power point conditions, while Figure 10 shows that the curve slope at MPP is precisely zero. Lastly, it is worth noticing that if you want to compare the proposed approach with the other ones available in the literature, we must take into account both the computational costs and the accuracy of results. Thus we have, on one hand, exact methods such as those proposed in [34, 46] which are efficient but cannot be implemented in low cost environments (i.e., they have significant computational costs) such as microcontroller based systems; on the other hand, approximated methods exist (see the one reported in [49]) which have low computational costs but introduce errors in the calculation of the parameters. On the contrary, the approach herein proposed is efficient from the computational cost point of view and accurate in the estimation of the five parameters: this is the best tradeoff currently obtained with respect to the other approaches presented in the literature.

\section{Conclusions}

A hybrid neural network approach based tool for identifying the PV one-diode model has been proposed. This method exploits the potentialities of the $\mathrm{NN}$ together with the effectiveness of the reduced form of one-diode model. The reduced form has been used on one hand to analyze the CEC database, which collects the datasheet parameters of about 10000 PV panels, and to build the training and test sets on the other hand as a part of the calculation procedure of the whole approach. Indeed, from the studies performed by the authors, it was found that a direct computation of the five parameters via multiple inputs and multiple outputs (MIMO) NN with acceptable computational resources is a prohibitive task. This also justifies why in the literature the neural network approach was often overlooked due to the obstacles encountered in particular in the prediction of 


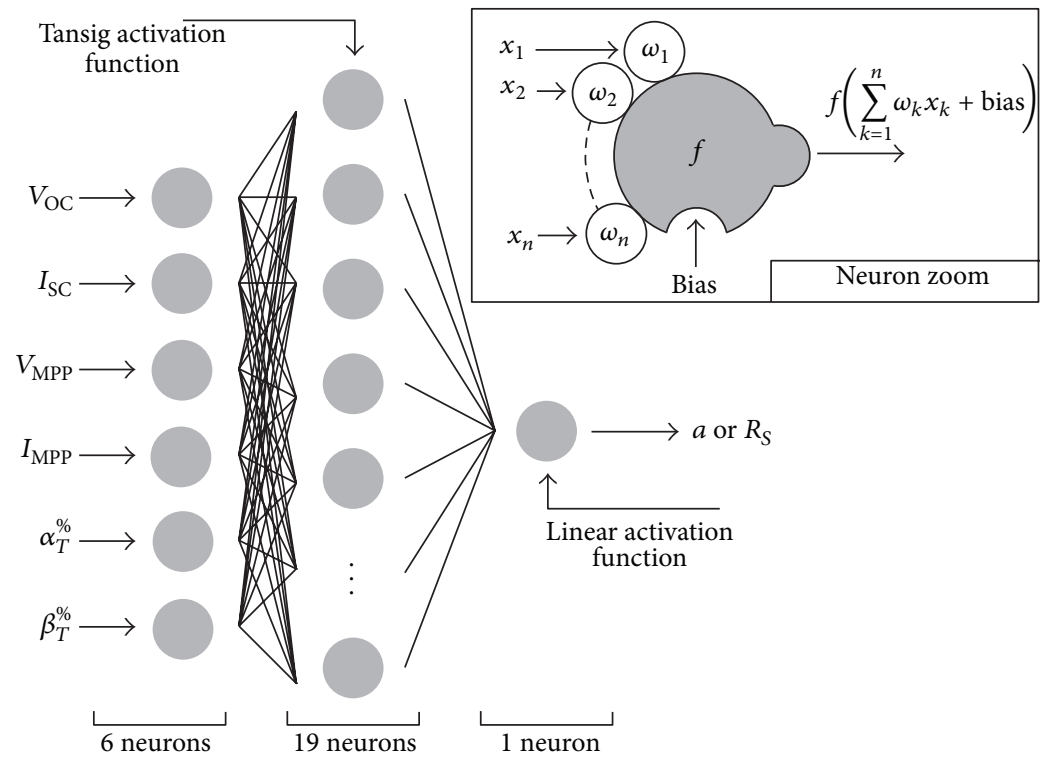

FIgURE 7: Architecture of the two adopted MLP NNs.

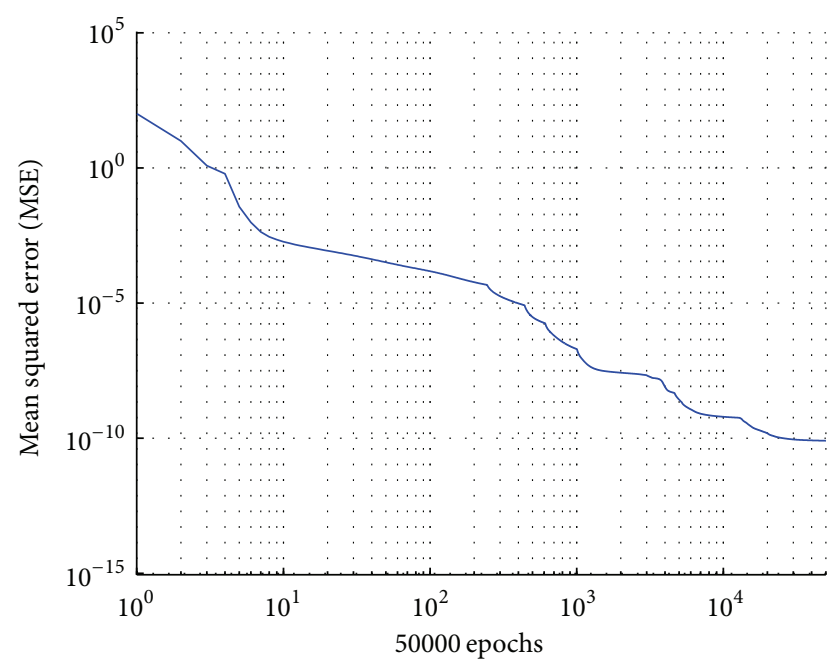

FIgure 8: Trend of the MSE during the training of the neural network with 19 hidden neurons and 50000 epochs tailored for predicting the $a$ parameter.

the shunt resistance. For this reason, in the presented approach, we adopted a NN predicting just two parameters, $R_{S}$ and $a$, together with the reduced form for the computation of the remaining three unknown parameters of the onediode model. The so achieved hybrid system constitutes a complete and extremely easy tool suitable to be implemented in a microcontroller based architecture. The obtained encouraging results prove that the proposed approach is efficient from the computational cost point of view and accurate in the estimation of the five parameters: the best currently existing tradeoff with respect to the approaches presented in the literature. The proposed method may be another important step, made thanks to soft computing techniques,

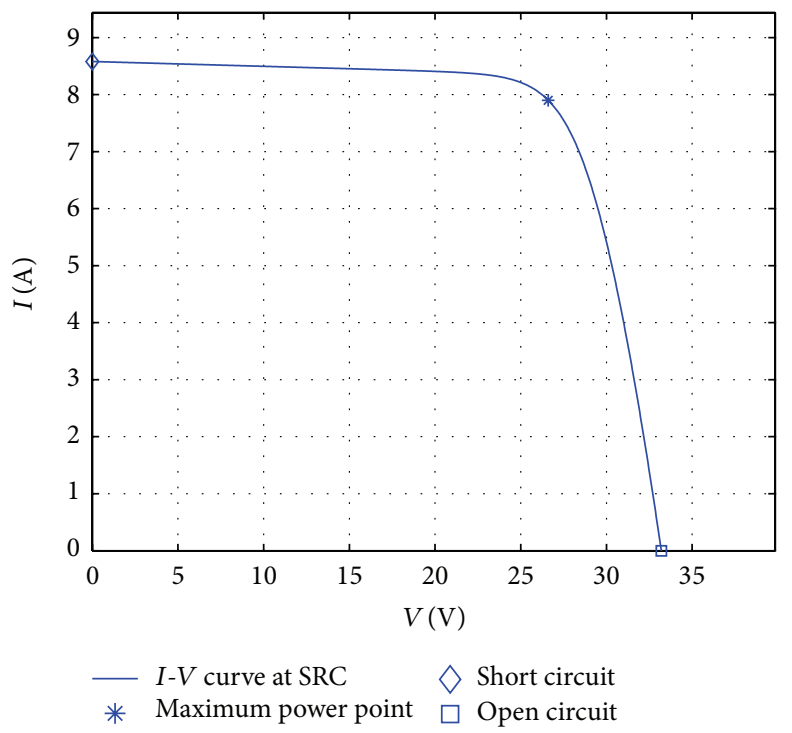

FIGURE 9: Graph of the $I-V$ curve for the PV module manufactured by Kyocera, Panel KD210GX LP, by using the obtained parameters.

in the development of intelligent systems for the monitoring and the management of renewable based generation plants.

\section{Nomenclature}

CEC: California Energy Commission

MIMO: Multiple input multiple output

MISO: Multiple input single output

mono-Si: Monocrystalline silicon

MLP: $\quad$ Multilayer perceptron

MPP: $\quad$ Maximum power point

MPPT: Maximum power point tracking

MSE: $\quad$ Mean squared error 


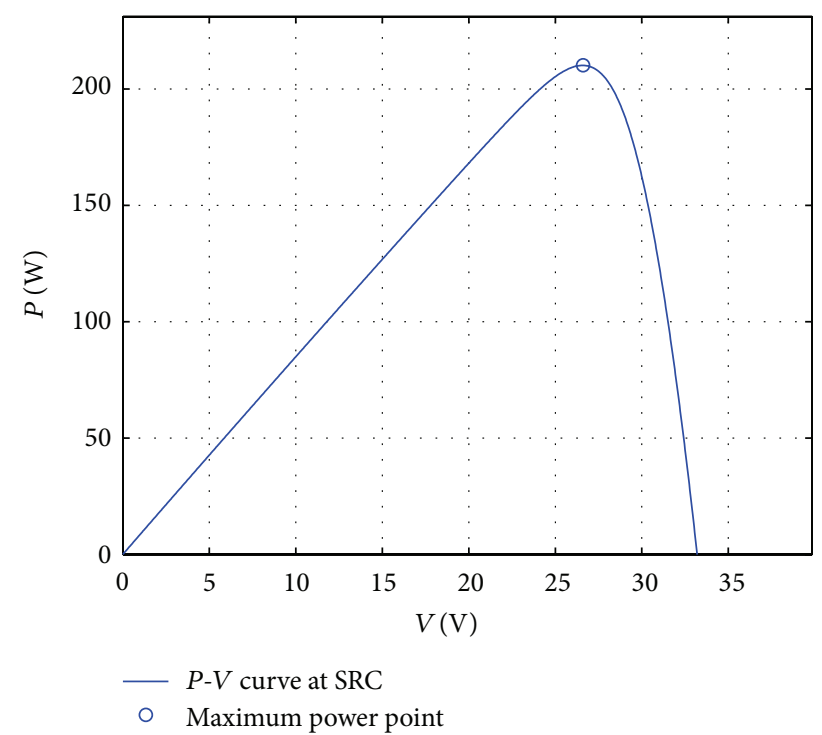

FIgURE 10: Graph of the $P-V$ curve for the PV module manufactured by Kyocera, Panel KD210GX LP, by using the obtained parameters.

multi-Si: Multicrystalline silicon

$\mathrm{NN}(\mathrm{s})$ : Neural network(s)

OC: $\quad$ Open circuit

PV: Photovoltaic

REF: $\quad$ Standard reference conditions

SRC: $\quad$ Standard reference conditions

SC: $\quad$ Short circuit

$S: \quad$ Irradiance

T: $\quad$ Cell temperature

$n: \quad$ Ideality factor

$N_{s}: \quad$ Number of series modules/cells

a: $\quad N_{S} n$

$T_{\text {ref: }}: \quad$ Temperature $25^{\circ} \mathrm{C}$ at $\mathrm{SRC}$

$S_{\text {ref }}: \quad$ Irradiance $1000 \mathrm{~W} / \mathrm{m}^{2}$ at SRC

$R_{S}: \quad$ Series resistance

$I_{\text {Irr: }} \quad$ Photocurrent

$I_{0}$ : $\quad$ Reverse saturation current

$R_{\mathrm{SH}}: \quad$ Shunt resistance

$G_{\mathrm{SH}}: \quad R_{\mathrm{SH}}^{-1}$

$V_{\mathrm{OC}}: \quad$ Open circuit voltage

$I_{\mathrm{SC}}: \quad$ Short circuit current

$V_{\text {MPP }}: \quad$ Maximum power voltage

$I_{\mathrm{MPP}}$ : Maximum power current

$\alpha_{T}^{\%}: \quad$ Temperature coefficient for $I_{\mathrm{SC}}$ in $\%$

$\beta_{T}^{\%}$ : $\quad$ Temperature coefficient for $V_{\mathrm{OC}}$ in $\%$.

\section{Conflict of Interests}

The authors declare that there is no conflict of interests regarding the publication of this paper.

\section{References}

[1] A. Luque and S. Hegedus, Handbook of Photovoltaic Science and Engineering, John Wiley \& Sons, New York, NY, USA, 2011.
[2] M. Carrasco, F. Mancilla-David, F. R. Fulginei, A. Laudani, and A. Salvini, "A neural networks-based maximum power point tracker with improved dynamics for variable dc-link gridconnected photo-voltaic power plants," International Journal of Applied Electromagnetics and Mechanics, vol. 43, no. 1-2, pp. 127135, 2013.

[3] K. M. El-Naggar, M. R. AlRashidi, M. F. AlHajri, and A. K. AlOthman, "Simulated Annealing algorithm for photovoltaic parameters identification," Solar Energy, vol. 86, no. 1, pp. 266274, 2012.

[4] M. Zagrouba, A. Sellami, M. Bouaïcha, and M. Ksouri, "Identification of PV solar cells and modules parameters using the genetic algorithms: application to maximum power extraction," Solar Energy, vol. 84, no. 5, pp. 860-866, 2010.

[5] K. Ishaque and Z. Salam, "An improved modeling method to determine the model parameters of photovoltaic (PV) modules using differential evolution (DE)," Solar Energy, vol. 85, no. 9, pp. 2349-2359, 2011.

[6] L. L. Jiang, D. L. Maskell, and J. C. Patra, "Parameter estimation of solar cells and modules using an improved adaptive differential evolution algorithm," Applied Energy, vol. 112, pp. 185-193, 2013.

[7] W. Peng, Y. Zeng, H. Gong, Y.-Q. Leng, Y.-H. Yan, and W. Hu, "Evolutionary algorithm and parameters extraction for dyesensitised solar cells one-diode equivalent circuit model," Micro \& Nano Letters, vol. 8, no. 2, pp. 86-89, 2013.

[8] M. U. Siddiqui and M. Abido, "Parameter estimation for five- and seven-parameter photovoltaic electrical models using evolutionary algorithms," Applied Soft Computing Journal, vol. 13, no. 12, pp. 4608-4621, 2013.

[9] A. Askarzadeh and A. Rezazadeh, "Artificial bee swarm optimization algorithm for parameters identification of solar cell models," Applied Energy, vol. 102, pp. 943-949, 2013.

[10] N. Rajasekar, N. Krishna Kumar, and R. Venugopalan, "Bacterial foraging algorithm based solar PV parameter estimation," Solar Energy, vol. 97, pp. 255-265, 2013.

[11] A. Orioli and A. Di Gangi, "A procedure to calculate the fiveparameter model of crystalline silicon photovoltaic modules on the basis of the tabular performance data," Applied Energy, vol. 102, pp. 1160-1177, 2013.

[12] P. Wolf and V. Benda, "Identification of PV solar cells and modules parameters by combining statistical and analytical methods," Solar Energy, vol. 93, pp. 151-157, 2013.

[13] S. Lineykin, M. Averbukh, and A. Kuperman, "An improved approach to extract the single-diode equivalent circuit parameters of a photovoltaic cell/panel," Renewable and Sustainable Energy Reviews, vol. 30, pp. 282-289, 2014.

[14] Y.-Y. Hong, F.-J. Lin, and F.-Y. Hsu, "Enhanced particle swarm optimization-based feeder reconfiguration considering uncertain large photovoltaic powers and demands," International Journal of Photoenergy, vol. 2014, Article ID 704839, 10 pages, 2014.

[15] Y.-H. Liu, S.-C. Huang, J.-W. Huang, and W.-C. Liang, "A particle swarm optimization-based maximum power point tracking algorithm for PV systems operating under partially shaded conditions," IEEE Transactions on Energy Conversion, vol. 27, no. 4, pp. 1027-1035, 2012.

[16] K. Ishaque, Z. Salam, A. Shamsudin, and M. Amjad, "A direct control based maximum power point tracking method for photovoltaic system under partial shading conditions using particle swarm optimization algorithm," Applied Energy, vol. 99, pp. 414-422, 2012. 
[17] S. Subiyanto, A. Mohamed, and M. A. Hannan, "Intelligent maximum power point tracking for PV system using hopfield neural network optimized fuzzy logic controller," Energy and Buildings, vol. 51, pp. 29-38, 2012.

[18] H. Mahamudul, M. Saad, and M. I. Henk, "Photovoltaic system modeling with fuzzy logic based maximum power point tracking algorithm," International Journal of Photoenergy, vol. 2013, Article ID 762946, 10 pages, 2013.

[19] S. Lalouni, D. Rekioua, T. Rekioua, and E. Matagne, "Fuzzy logic control of stand-alone photovoltaic system with battery storage," Journal of Power Sources, vol. 193, no. 2, pp. 899-907, 2009.

[20] R. S. Boata and P. Gravila, "Functional fuzzy approach for forecasting daily global solar irradiation," Atmospheric Research, vol. 112, pp. 79-88, 2012.

[21] C. Ze, Z. Pang, Y. Liu, and P. Xue, "An adaptive solar photovoltaic array reconfiguration method based on fuzzy control," in Proceedings of the 8th World Congress on Intelligent Control and Automation (WCICA '10), pp. 176-181, Jinan, China, July 2010.

[22] S. A. Kalogirou, "Artificial neural networks in renewable energy systems applications: a review," Renewable and Sustainable Energy Reviews, vol. 5, no. 4, pp. 373-401, 2000.

[23] A. K. Mahamad and S. Saon, "Development of artificial neural network based MPPT for photovoltaic system during shading condition," Applied Mechanics and Materials, vol. 448-453, pp. 1573-1578, 2014.

[24] M. R. Vincheh, A. Kargar, and G. A. Markadeh, "A hybrid control method for maximum power point tracking (MPPT) in photovoltaic systems," Arabian Journal for Science and Engineering, vol. 39, no. 6, pp. 4715-4725, 2014.

[25] Y.-H. Liu, C.-L. Liu, J.-W. Huang, and J.-H. Chen, "Neuralnetwork-based maximum power point tracking methods for photovoltaic systems operating under fast changing environments," Solar Energy, vol. 89, pp. 42-53, 2013.

[26] K. Punitha, D. Devaraj, and S. Sakthivel, "Artificial neural network based modified incremental conductance algorithm for maximum power point tracking in photovoltaic system under partial shading conditions," Energy, vol. 62, pp. 330-340, 2013.

[27] A. K. Yadav and S. S. Chandel, "Solar radiation prediction using artificial neural network techniques: a review," Renewable \& Sustainable Energy Reviews, vol. 33, pp. 772-781, 2014.

[28] B. M. Wilamowski, D. Hunter, and A. Malinowski, "Solving parity-n problems with feedforward neural networks," in Proceedings of the International Joint Conference on Neural Networks, vol. 4, pp. 2546-2551, IEEE, July 2003.

[29] G. Capizzi, S. Coco, C. Giuffrida, and A. Laudani, "A neural network approach for the differentiation of numerical solutions of 3-D electromagnetic problems," IEEE Transactions on Magnetics, vol. 40, no. 2, pp. 953-956, 2004.

[30] C. Xu, C. Wang, F. Ji, and X. Yuan, "Finite-element neural network-based solving 3-D differential equations in mfl," IEEE Transactions on Magnetics, vol. 48, no. 12, pp. 4747-4756, 2012.

[31] N. J. Blair, A. P. Dobos, and P. Gilman, "Comparison of photovoltaic models in the system advisor model," in Proceedings of the National Solar Conference (SOLAR '13), Baltimore, Md, USA, April 2010.

[32] A. Laudani, F. R. Fulginei, and A. Salvini, "Identification of the one-diode model for photovoltaic modules from datasheet values," Solar Energy, vol. 108, pp. 432-446, 2014.
[33] W. de Soto, S. A. Klein, and W. A. Beckman, "Improvement and validation of a model for photovoltaic array performance," Solar Energy, vol. 80, no. 1, pp. 78-88, 2006.

[34] A. Laudani, F. Riganti Fulginei, A. Salvini, G. M. Lozito, and S. Coco, "Very fast and accurate procedure for the characterization of photovoltaic panels from datasheet information," International Journal of Photoenergy, vol. 2014, Article ID 946360, 10 pages, 2014.

[35] A. Laudani, F. Mancilla-David, F. Riganti-Fulginei, and A. Salvini, "Reduced-form of the photovoltaic five-parameter model for efficient computation of parameters," Solar Energy, vol. 97, pp. 122-127, 2013.

[36] A. Laudani, F. R. Fulginei, and A. Salvini, "High performing extraction procedure for the one-diode model of a photovoltaic panel from experimental I-V curves by using reduced forms," Solar Energy, vol. 103, pp. 316-326, 2014.

[37] California Energy Commission, "CECPV calculator version 4.0," 2013, http://www.gosolarcalifornia.org/tools/nshpcalculator/.

[38] G. Cybenko, "Approximation by superpositions of a sigmoidal function," Mathematics of Control, Signals, and Systems, vol. 2, no. 4, pp. 303-314, 1989.

[39] K. Hornik, M. Stinchcombe, and H. White, "Multilayer feedforward networks are universal approximators," Neural Networks, vol. 2, no. 5, pp. 359-366, 1989.

[40] J. Ilonen, J.-K. Kamarainen, and J. Lampinen, "Differential evolution training algorithm for feed-forward neural networks," Neural Processing Letters, vol. 17, no. 1, pp. 93-105, 2003.

[41] R. Rawat, J. K. Patel, and M. T. Manry, "Minimizing validation error with respect to network size and number of training epochs," in Proceedings of the International Joint Conference on Neural Networks (IJCNN '13), pp. 1-7, Dallas, Tex, USA, August 2013.

[42] D. Hunter, H. Yu, M. S. Pukish III, J. Kolbusz, and B. M. Wilamowski, "Selection of proper neural network sizes and architectures-a comparative study," IEEE Transactions on Industrial Informatics, vol. 8, no. 2, pp. 228-240, 2012.

[43] E. J. Teoh, K. C. Tan, and C. Xiang, "Estimating the number of hidden neurons in a feedforward network using the singular value decomposition," IEEE Transactions on Neural Networks, vol. 17, no. 6, pp. 1623-1629, 2006.

[44] C.-T. Kim and J.-J. Lee, "Training two-layered feedforward networks with variable projection method," IEEE Transactions on Neural Networks, vol. 19, no. 2, pp. 371-375, 2008.

[45] B. M. Wilamowski, "Neural network architectures and learning algorithms," IEEE Industrial Electronics Magazine, vol. 3, no. 4, pp. 56-63, 2009.

[46] F. Mancilla-David, F. Riganti-Fulginei, A. Laudani, and A. Salvini, "A neural network-based low-cost solar irradiance sensor," IEEE Transactions on Instrumentation and Measurement, vol. 63, no. 3, pp. 583-591, 2014.

[47] F. R. Fulginei, A. Salvini, and M. Parodi, "Learning optimization of neural networks used for MIMO applications based on multivariate functions decomposition," Inverse Problems in Science and Engineering, vol. 20, no. 1, pp. 29-39, 2012.

[48] F. R. Fulginei, A. Laudani, A. Salvini, and M. Parodi, "Automatic and parallel optimized learning for neural networks performing MIMO applications," Advances in Electrical and Computer Engineering, vol. 13, no. 1, pp. 3-12, 2013.

[49] J. Cubas, S. Pindado, and M. Victoria, "On the analytical approach for modeling photovoltaic systems behavior," Journal of Power Sources, vol. 247, pp. 467-474, 2014. 
[50] Y. Li, W. Huang, H. Huang et al., "Evaluation of methods to extract parameters from current-voltage characteristics of solar cells," Solar Energy, vol. 90, pp. 51-57, 2013. 

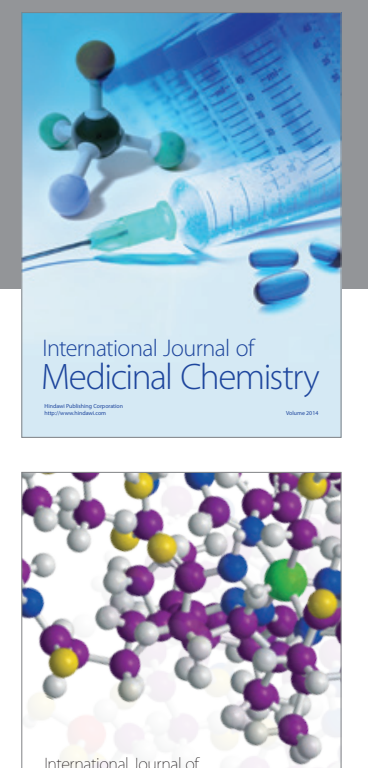

\section{Carbohydrate} Chemistry

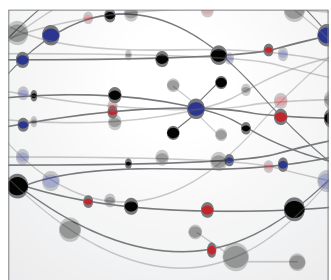

The Scientific World Journal
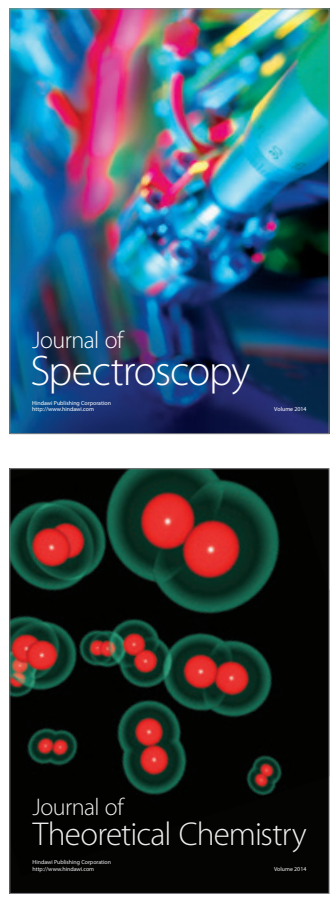
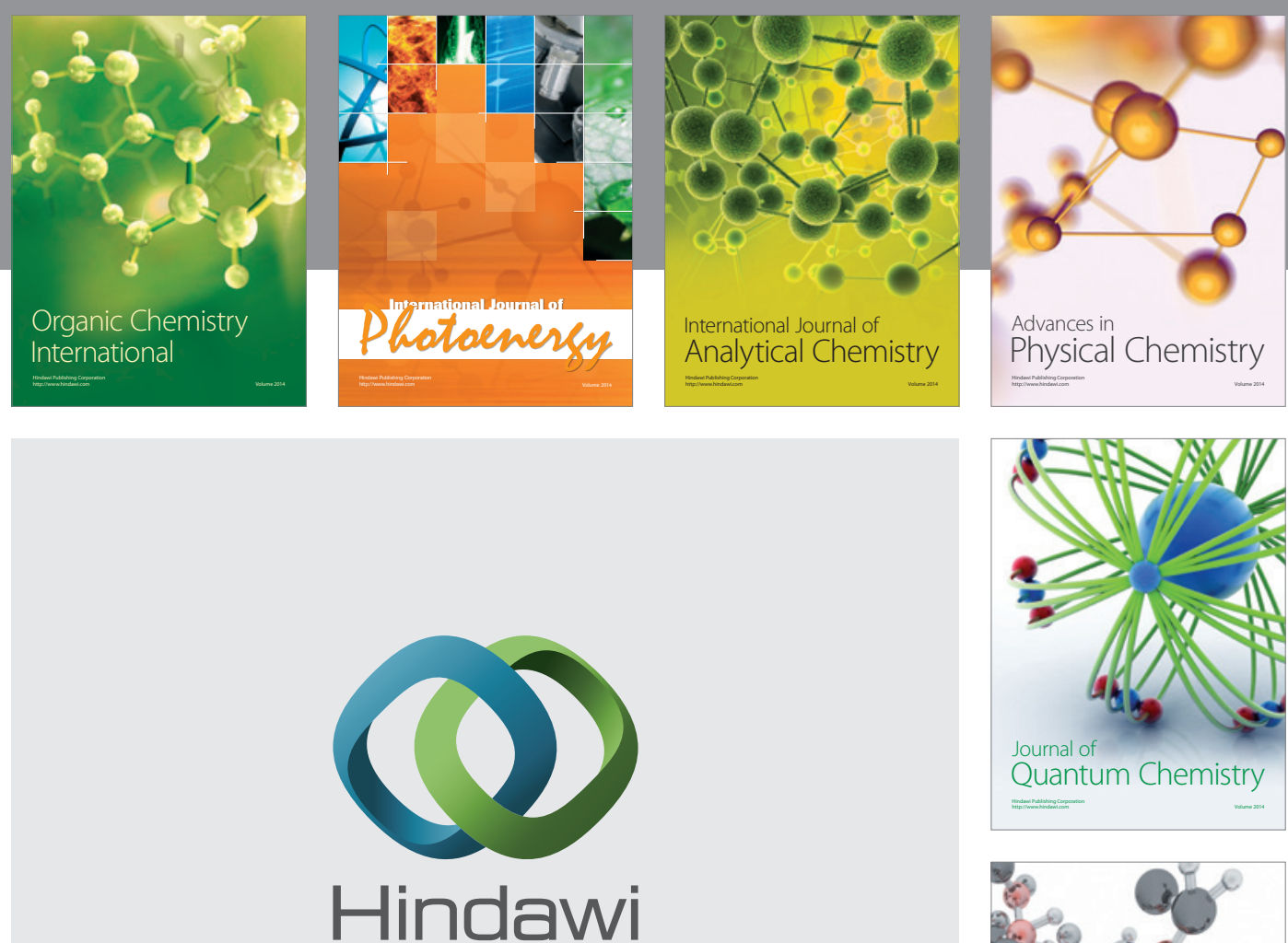

Submit your manuscripts at

http://www.hindawi.com

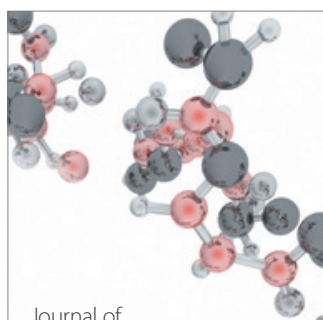

Analytical Methods

in Chemistry

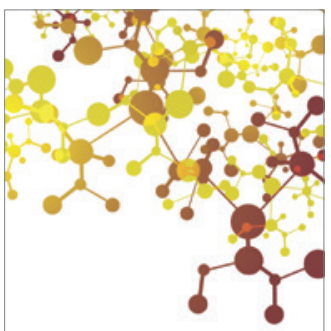

Journal of

Applied Chemistry

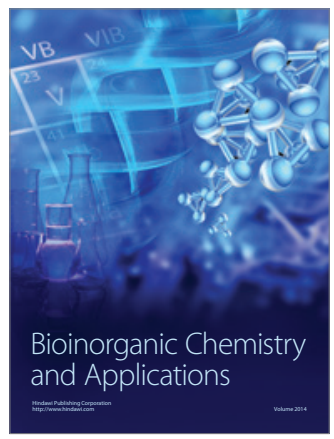

Inorganic Chemistry
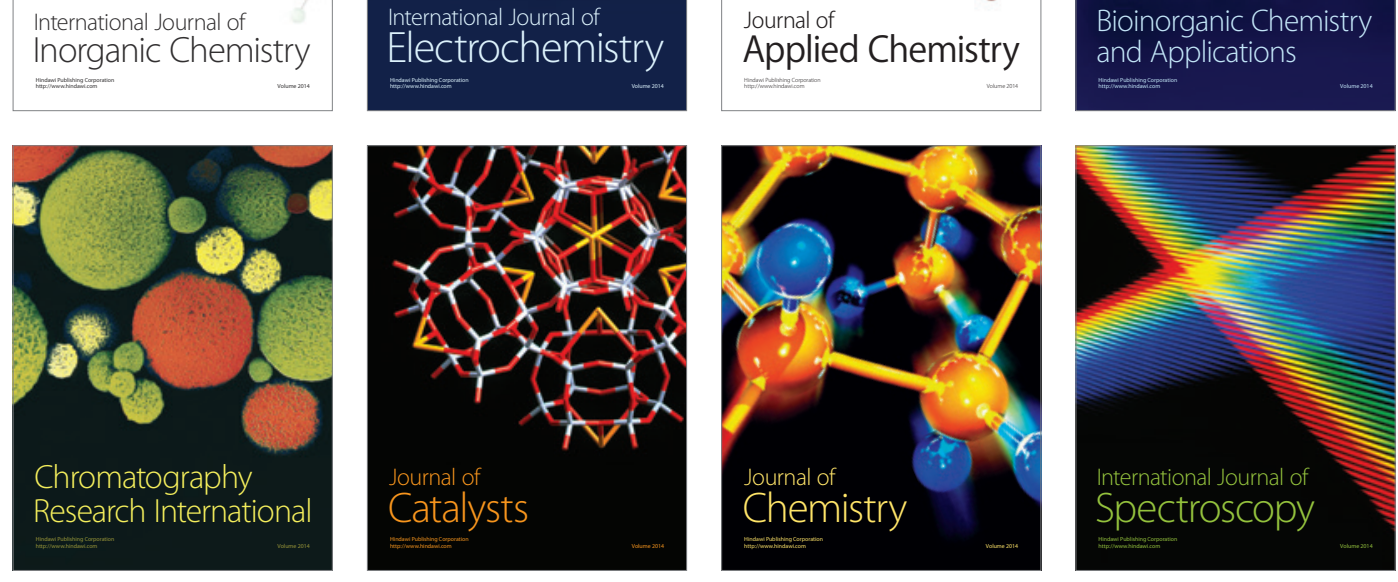